\title{
Constant modulus algorithm aided soft decision directed scheme for blind space-time equalisation of SIMO channels ${ }^{\text {tr }}$
}

\author{
S. Chen*, A. Wolfgang, L. Hanzo \\ School of Electronics and Computer Science, University of Southampton, Highfield, Southampton SO17 1BJ, UK
}

Received 27 September 2006; received in revised form 23 January 2007; accepted 17 April 2007

Available online 22 April 2007

\begin{abstract}
This paper investigates a blind space-time equaliser (STE) designed for single-input multiple-output (SIMO) systems that employ high-throughput quadrature amplitude modulation schemes. A constant modulus algorithm (CMA) aided soft decision-directed (SDD) scheme, originally derived for low-complexity blind equalisation of single-input single-output channels, is extended to the SIMO scenario. Simulations are conducted to compare the performance of this blind adaptive scheme with another low-complexity blind STE referred to as the CMA aided decision directed (DD) scheme. The results obtained demonstrate that for SIMO systems the CMA aided SDD scheme exhibits advantages over the CMA aided DD arrangement, in terms of its faster convergence speed and lower computational complexity.
\end{abstract}

(C) 2007 Elsevier B.V. All rights reserved.

Keywords: Single-input multiple-output; Space-time equalisation; Quadrature amplitude modulation; Constant modulus algorithm; Decision-directed adaptation

\section{Introduction}

Space-time processing techniques have increasingly been applied to wireless communication systems [1-6]. With the aid of smart antenna arrays and by exploiting both the space and time dimensions, space-time processing is capable of effectively improving the achievable system capacity, coverage and quality of service by both suppressing intersymbol interference and co-channel

\footnotetext{
The financial support of the EU under the auspices of the Phoenix project is gratefully acknowledged.

*Corresponding author. Tel.: + 442380596660 ; fax: +442380594508 .

E-mail addresses: sqc@ecs.soton.ac.uk (S. Chen), aw03r@ecs.soton.ac.uk (A. Wolfgang), lh@ecs.soton.ac.uk (L. Hanzo).
}

interference, i.e. interference cancellation. The family of single-input multiple-output (SIMO) systems has enjoyed popularity owing to its simplicity. A SIMO system consists of a single-antenna transmitter and a receiver equipped with multiple antennas. A space-time equaliser (STE) based on this SIMO structure is capable of mitigating the channel impairment arising from hostile multipath propagation. For the sake of improving the achievable bandwidth efficiency, high-throughput quadrature amplitude modulation (QAM) [7] schemes have become popular in numerous wireless network standards. For the sake of further improving the achievable system throughput, invoking blind adaptation aided STE is attractive, as this avoids the reduction of the effective throughput by invoking training. 
For single transmitter antenna and single receiver antenna (SISO) systems with high-order QAM schemes, the constant modulus algorithm (CMA)based blind equaliser constitutes by far the most popular design option [8-11], due to its low computational complexity. The steady-state performance of the CMA in terms of its mean square error (MSE), however, may not be sufficiently low for the system to achieve an adequate bit error ratio performance. A standard solution is to switch to a decision directed (DD) adaptation to further minimise the CMA's steady-state MSE [12]. However, in order for the DD adaptation to become successful, the CMA's steady-state MSE should already be sufficiently low, and in practice such a low level of MSE may not always be achievable by the CMA [13]. An alternative is to adopt a composite cost function, which consists of an amalgam of the CMA's blind adaptation cost function and the decision-based MSE [14]. The weighting of the two component cost functions, however, must be carefully chosen. The first soft decision directed (SDD) blind equalisation algorithm that maximises the marginal probability density function (PDF) of the equaliser's output was derived in [15] for binary phase shift keying modulation. It was extended to quadrature phase shiftkeying modulation in [16]. Chen and his co-workers [17] developed a multistage adaptive scheme to extend this SDD blind equaliser to high-order QAM schemes, without substantially increasing the computational complexity imposed.

De Castro and his co-workers [13] have proposed an attractive blind adaptive scheme for conventional SISO arrangements. Rather than switching to DD adaptation after the CMA has converged or adopting the composite CMA and decision-based MSE cost function, their scheme consists of a DD equaliser operating in unison with a CMA-based equaliser. To avoid error propagation owing to incorrect decisions, the DD weight adaptation only takes place, if the CMA's adaptation is deemed to have achieved a successful adjustment of the equaliser weight vector with a high probability. At a cost of slightly more than doubling the complexity of the simple CMA, this combined CMA and DD equaliser was reported to achieve a dramatic equalisation performance improvement over the CMA [13]. Recently, a novel combined CMA and SDD blind equaliser has been proposed [18], which was shown to have a faster convergence speed and simpler implementation than the combined CMA and DD scheme. This combined CMA and SDD equaliser operates a CMA equaliser and a last-stage SDD equaliser of [17] in a truly parallel fashion.

Recent research on blind adaptive SIMO systems has been focused on a number of topics, including blind channel estimation [19-21]. The decoupled weighted iterative least squares algorithm with projection (DW-ILSP) [22,23], which is a batch expectation maximisation (EM)-type blind algorithm, has also been proposed for multiple-receiver-antenna aided systems communicating over flat-fading channels using 4-QAM [22]. For high-order QAM signalling over frequency-selective channels, however, the complexity of the blind DW-ILSP algorithm may become prohibitive. In the context of blind beamforming, the CMA-type blind adaptive algorithm has been used before in [24-27]. However, the combined CMA and SDD (or DD) scheme has not been employed for blind adaptive SIMO systems, especially not in conjunction with high-order QAM schemes.

Against this backdrop, in this paper we extend the low-complexity CMA, the CMA + DD, and the CMA + SDD blind adaptive arrangements, which were originally derived for SISO systems, to a novel blind STE for employment in SIMO systems. The simulation results obtained demonstrate that the CMA + DD and CMA + SDD algorithms achieve a similar steady-state equalisation performance, both resulting in a dramatic improvement over the performance of the pure CMA STE. However, the CMA + SDD aided blind STE has a faster convergence and imposes a lower computational complexity in comparison to the CMA + DD scheme, as it was also observed in the case of SISO systems. This study clearly demonstrates that the combined CMA and SDD algorithm constitutes an attractive blind STE for employment in SIMO systems using high-throughput QAM schemes.

\section{System model}

The SIMO system employing a single transmitter and $L(>1)$ receiver antennas is depicted in Fig. 1, where $s(t)$ is the transmitted signal, $x_{l}(t)$ denotes the $l$ th receiver antenna's output signal and $e_{l}(t)$ the $l$ th channel's noise. The received signals are sampled at a rate higher than the symbol rate $f_{\mathrm{s}}$ to obtain the $L$ antennas' output signals $\bar{x}_{l}(n), 1 \leqslant l \leqslant L$, which are passed to a fractionally spaced (FS) STE, as shown in Fig. 2. FS equalisation offers many advantages over symbol-spaced one [28], including better 
equalisation performance, lower sensitivity to timing error and faster convergence speed. The last property is valid provided that the length of the equaliser is not excessively long. Since all these advantages are possessed by a $T_{\mathrm{s}} / 2$-spaced sampling, where $T_{\mathrm{s}}$ is the symbol period, the sampling rate is taken to be $2 f_{\mathrm{s}}$ and the delay unit $\Delta$ in the temporal filters equals $T_{\mathrm{s}} / 2$. For notational convenience, the index $k$ is reserved for $T_{\mathrm{s}}$-spaced sampled quantities, while the index $n$ for $T_{\mathrm{s}} / 2$ spaced sampled quantities. The transmitted $T_{\mathrm{s}^{-}}$ spaced symbol sequence $s(k)=s_{R}(k)+j s_{I}(k)$ takes

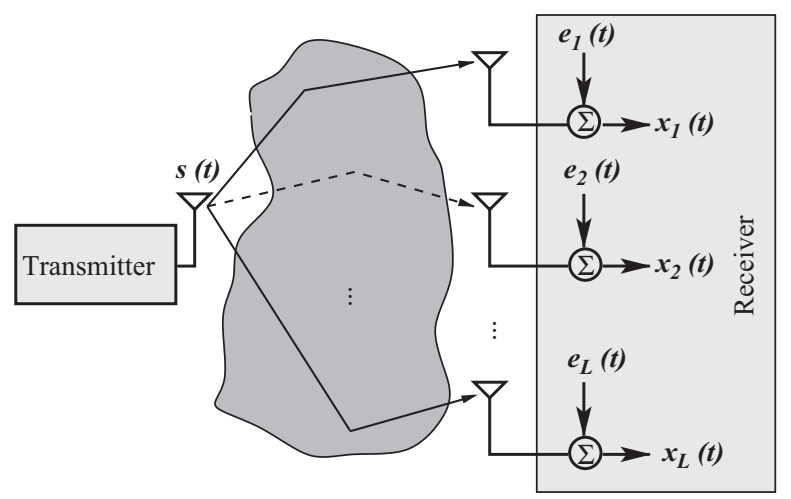

Fig. 1. Single-input multiple-output system employing multiple receiver antennas. the values from the $M$-QAM symbol set defined by $\mathscr{S}=\left\{s_{i, l}=(2 i-Q-1)+j(2 l-Q-1), 1 \leqslant i, l \leqslant Q\right\}$,

where $Q=\sqrt{M}$ and $j=\sqrt{-1}$. The $T_{\mathrm{s}} / 2$-spaced signal sample $\bar{x}_{l}(n)$ of the $l$ th antenna is given by

$\bar{x}_{l}(n)=\sum_{i=0}^{2 N_{c}-1} \bar{a}_{i, l} \bar{s}(n-i)+\bar{e}_{l}(n)$,

where the $T_{\mathrm{s}} / 2$-spaced sequence $\{\bar{s}(n)\}$ is a zeropadded version of the transmitted symbol sequence $\{s(k)\}$ :

$\bar{s}(n)= \begin{cases}s(n / 2) & \text { for even } n, \\ 0 & \text { for odd } n .\end{cases}$

The $T_{\mathrm{s}} / 2$-spaced channel impulse response (CIR) of the $l$ th channel is $\overline{\mathbf{a}}_{l}=\left[\begin{array}{lllll}\bar{a}_{0, l} & \bar{a}_{1, l} & \bar{a}_{2, l} & \bar{a}_{3, l} & \cdots\end{array}\right.$ $\left.\bar{a}_{2 N_{c}-1, l}\right]^{\mathrm{T}}$, with $N_{c}$ corresponding to the $l$ th $T_{\mathrm{s}}$-spaced CIR length, and the $T_{\mathrm{s}} / 2$-spaced noise sample $\bar{e}_{l}(n)=\bar{e}_{l, R}(n)+j \bar{e}_{l, I}(n)$ is a complex-valued Gaussian white noise with $E\left[\bar{e}_{l, R}^{2}(n)\right]=E\left[\bar{e}_{l, I}^{2}(n)\right]=\sigma_{e}^{2}$.

The STE consists of $L T_{\mathrm{s}} / 2$-spaced temporal filters, and its output is defined by

$$
\bar{y}(n)=\sum_{l=1}^{L} \sum_{i=0}^{2 m-1} \bar{w}_{i, l} \bar{x}_{l}(n-i)=\sum_{l=1}^{L} \overline{\mathbf{w}}_{l}^{\mathrm{T}} \overline{\mathbf{x}}_{l}(n)=\overline{\mathbf{w}}^{\mathrm{T}} \overline{\mathbf{x}}(n),
$$

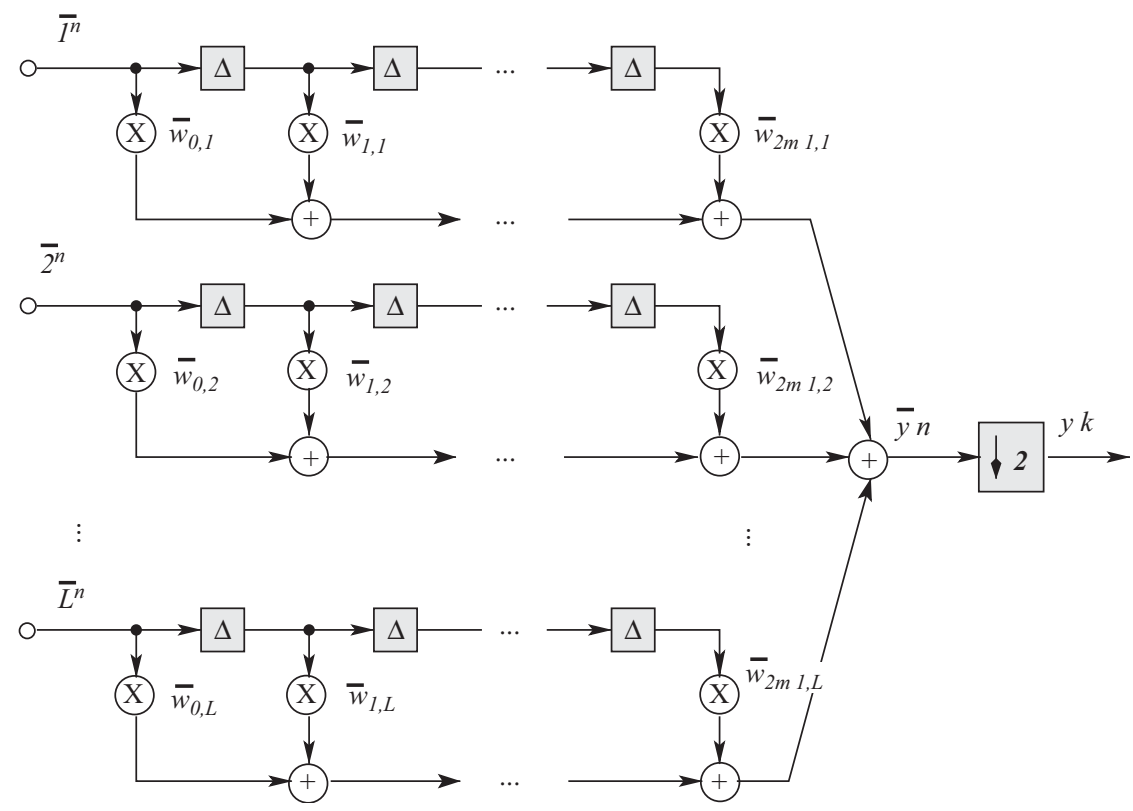

Fig. 2. Space-time equalisation structure using $\Delta$-spaced temporal filters, where $\Delta=T_{\mathrm{s}} / 2$ and $T_{\mathrm{s}}$ denotes the symbol period, the index $k$ indicates $T_{\mathrm{s}}$-spaced sampled quantities and index $n T_{\mathrm{s}} / 2$-spaced sampled quantities. 
where $2 m$ is the length of the $T_{\mathrm{s}} / 2$-spaced temporal filters, while

$\overline{\mathbf{w}}=\left[\begin{array}{llll}\overline{\mathbf{w}}_{1}^{\mathrm{T}} & \overline{\mathbf{w}}_{2}^{\mathrm{T}} & \cdots & \overline{\mathbf{w}}_{L}^{\mathrm{T}}\end{array}\right]^{\mathrm{T}}$

and

$\overline{\mathbf{x}}(n)=\left[\begin{array}{llll}\overline{\mathbf{x}}_{1}^{\mathrm{T}}(n) & \overline{\mathbf{x}}_{2}^{\mathrm{T}}(n) & \cdots & \overline{\mathbf{x}}_{L}^{\mathrm{T}}(n)\end{array}\right]^{\mathrm{T}}$

with $\overline{\mathbf{w}}_{l}=\left[\begin{array}{llll}\bar{w}_{0, l} & \bar{w}_{1, l} & \cdots & \bar{w}_{2 m-1, l}\end{array}\right]^{\mathrm{T}}$ being the complex-valued weight vector of the $l$ th temporal filter and $\quad \overline{\mathbf{x}}_{l}(n)=\left[\begin{array}{llll}\bar{x}_{l}(n) & \bar{x}_{l}(n-1) & \cdots & \bar{x}_{l}(n-2 m+1)\end{array}\right]^{\mathrm{T}}$ being the input vector of the $l$ th temporal filter. The $T_{\mathrm{s}} / 2$-spaced output $\bar{y}(n)$ is decimated by a factor of 2 to create the $T_{\mathrm{s}}$-spaced output $y(k)$. Following the approach [28], an equivalent $T_{\mathrm{s}}$ spaced model for $y(k)$ is used. Let us first define the following variables:

$\begin{aligned} \overline{\mathbf{a}}_{l}^{\mathrm{e}} & =\left[\begin{array}{llll}\bar{a}_{0, l} & \bar{a}_{2, l} & \cdots & \bar{a}_{2 N_{c}-2, l}\end{array}\right]^{\mathrm{T}}, \\ \overline{\mathbf{a}}_{l}^{\mathrm{o}} & =\left[\begin{array}{llll}\bar{a}_{1, l} & \bar{a}_{3, l} & \cdots & \bar{a}_{2 N_{c}-1, l}\end{array}\right]^{\mathrm{T}}, \\ \overline{\mathbf{w}}_{l}^{\mathrm{e}} & =\left[\begin{array}{llll}\bar{w}_{0, l} & \bar{w}_{2, l} & \cdots & \bar{w}_{2 m-2, l}\end{array}\right]^{\mathrm{T}}, \\ \overline{\mathbf{w}}_{l}^{\mathrm{o}} & =\left[\begin{array}{llll}\bar{w}_{1, l} & \bar{w}_{3, l} & \cdots & \bar{w}_{2 m-1, l}\end{array}\right]^{\mathrm{T}},\end{aligned}$

and

$e_{l}^{\mathrm{e}}(k)=\bar{e}_{l}(2 n), \quad e_{l}^{\mathrm{o}}(k)=\bar{e}_{l}(2 n+1)$,

$x_{l}^{\mathrm{e}}(k)=\bar{x}_{l}(2 n), \quad x_{l}^{\mathrm{o}}(k)=\bar{x}_{l}(2 n+1)$.

Note that $e_{l}^{\mathrm{e}}(k)$ and $e_{l}^{\mathrm{o}}(k)$ are even and odd noise samples, $x_{l}^{\mathrm{e}}(k)$ and $x_{l}^{\mathrm{o}}(k)$ are even and odd channel output samples, $\overline{\mathbf{a}}_{l}^{\mathrm{e}}$ and $\overline{\mathbf{a}}_{l}^{o}$ are even and odd channel tap vectors, and $\overline{\mathbf{w}}_{l}^{\mathrm{e}}$ and $\overline{\mathbf{w}}_{l}^{\mathrm{o}}$ are even and odd equaliser weight vectors, respectively, for the $l$ th channel. Furthermore, let us define

$$
\mathbf{w}_{l}=\left[\begin{array}{llll}
w_{0, l} & w_{1, l} & \cdots & w_{2 m-1, l}
\end{array}\right]^{\mathrm{T}}=\left[\begin{array}{lll}
\left(\overline{\mathbf{w}}_{l}^{\mathrm{o}}\right)^{\mathrm{T}} & \left(\overline{\mathbf{w}}_{l}^{\mathrm{e}}\right)^{\mathrm{T}}
\end{array}\right]^{\mathrm{T}}
$$

$$
\begin{aligned}
\mathbf{x}_{l}(k) & =\left[\begin{array}{llll}
x_{l}(k) & x_{l}(k-1) & \cdots & x_{l}(k-2 m+1)
\end{array}\right]^{\mathrm{T}} \\
& =\left[\begin{array}{lll}
\left(\mathbf{x}_{l}^{\mathrm{e}}(k)\right)^{\mathrm{T}} & \left(\mathbf{x}_{l}^{\mathrm{o}}(k)\right)^{\mathrm{T}}
\end{array}\right]^{\mathrm{T}},
\end{aligned}
$$

with $\quad \mathbf{x}_{l}^{\mathrm{e}}(k)=\left[\begin{array}{llll}x_{l}^{\mathrm{e}}(k) & x_{l}^{\mathrm{e}}(k-1) & \cdots & x_{l}^{\mathrm{e}}(k-m+1)\end{array}\right]^{\mathrm{T}}$ and $\quad \mathbf{x}_{l}^{\mathrm{o}}(k)=\left[\begin{array}{llll}x_{l}^{\mathrm{o}}(k) & x_{l}^{\mathrm{o}}(k-1) & \cdots & x_{l}^{\mathrm{o}}(k-m+1)\end{array}\right]^{\mathrm{T}}$. Then the $T_{\mathrm{s}}$-spaced STE output $y(k)$ is given by

$y(k)=\sum_{l=1}^{L} \sum_{i=0}^{2 m-1} w_{i, l} x_{l}(k-i)=\sum_{l=1}^{L} \mathbf{w}_{l}^{\mathrm{T}} \mathbf{x}_{l}(k)=\mathbf{w}^{\mathrm{T}} \mathbf{x}(k)$,

where $\mathbf{w}=\left[\begin{array}{llll}\mathbf{w}_{1}^{\mathrm{T}} & \mathbf{w}_{2}^{\mathrm{T}} & \cdots & \mathbf{w}_{L}^{\mathrm{T}}\end{array}\right]^{\mathrm{T}}$ and $\mathbf{x}(k)=\left[\begin{array}{lll}\mathbf{x}_{1}^{\mathrm{T}}(k) & \mathbf{x}_{2}^{\mathrm{T}}\end{array}\right.$ (k) $\left.\cdots \mathbf{x}_{L}^{\mathrm{T}}(k)\right]^{\mathrm{T}}$. We should define the complete system's signal to noise ratio (SNR) for the SIMO system under consideration as

$\mathrm{SNR}=\frac{\sigma_{s}^{2} \sum_{l=1}^{L} \sum_{i=0}^{2 N_{c}-1}\left|\bar{a}_{i, l}\right|^{2}}{4 L \sigma_{e}^{2}}$,

where $\sigma_{s}^{2}=E\left[|s(k)|^{2}\right]$. A commonly used initialisation strategy for SISO blind equalisation is to set the middle two taps of the $T_{\mathrm{s}} / 2$-spaced blind equaliser to $1+j 0$ and rest of the taps to $0+j 0$ [28]. We will extend this initialisation strategy to the present blind STE. Before blind adaptation, the STE's weights are initialised to $\bar{w}_{i, l}=1 / L+j 0$ for $i=$ $m-1$ and $m$, and $\bar{w}_{i, l}=0+j 0$ for all the other values of $i$, where $1 \leqslant l \leqslant L$.

\section{Low complexity blind adaptive algorithms for STE}

With the aid of the STE model (11), a number of low-complexity blind adaptive schemes originally developed for the SISO system can readily be extended to blind STE applications for SIMO systems.

\section{1. $C M A$}

Given the STE output $y(k)=\mathbf{w}^{\mathrm{T}}(k) \mathbf{x}(k)$ at the $T_{\mathrm{s}^{-}}$ spaced sample $k$, the CMA adjusts $\mathbf{w}$ according to

$\left.\begin{array}{l}\varepsilon(k)=y(k)\left(R^{2}-|y(k)|^{2}\right) \\ \mathbf{w}(k+1)=\mathbf{w}(k)+\mu_{\mathrm{CMA}} \varepsilon(k) \mathbf{x}^{*}(k)\end{array}\right\}$,

where $R^{2}$ is a positive constant defined by $R^{2}=E\left[|s(k)|^{4}\right] / E\left[|s(k)|^{2}\right], \mu_{\mathrm{CMA}}$ is an adaptive gain and $\mathbf{x}^{*}(k)$ is the complex conjugate of $\mathbf{x}(k)$. Typically, a very small $\mu_{\mathrm{CMA}}$ has to be used for ensuring convergence. The CMA has a very low computational complexity, as summarised in Table 1. The CMA does not require the knowledge of the symbol constellation. It is also robust to imperfect carrier recovery and capable of opening "initially closed eye" at the STE's output. A wellknown problem of the CMA is that its steady-state MSE may not be sufficiently low for ensuring an adequate bit error rate performance.

\subsection{Combined CMA and DD scheme}

Let the weight vector of the STE be split into two parts, yielding $\mathbf{w}=\mathbf{w}_{\mathrm{c}}+\mathbf{w}_{\mathrm{d}}$. Specifically $\mathbf{w}_{\mathrm{c}}$ is the weight vector of the CMA STE, which is designed for minimising the CMA cost function, while $\mathbf{w}_{\mathrm{d}}$ is the weight vector of the DD STE, which is designed 
Table 1

Comparison of computational complexity per weight update

\begin{tabular}{llll}
\hline Equaliser & Multiplications & Additions & e \\
\hline CMA & $8 \times(L \times 2 \mathrm{~m})+6$ & $8 \times(L \times 2 \mathrm{~m})+2 \times L-2$ & - \\
CMA + DD & $16 \times(L \times 2 \mathrm{~m})+8$ & $20 \times(L \times 2 \mathrm{~m})+4 \times L-4$ & - \\
CMA + SDD & $12 \times(L \times 2 \mathrm{~m})+29$ & $14 \times(L \times 2 \mathrm{~m})+2 \times L+19$ & 4
\end{tabular}

The number of receiver antennas is $L$ and the length of temporal filters is $2 \mathrm{~m}$.

for minimising the decision-based MSE:

$\bar{J}_{\mathrm{DD}}(\mathbf{w})=\frac{1}{2} E\left[|\mathscr{2}[y(k)]-y(k)|^{2}\right]$,

where $2[y(k)]$ denotes the quantised equaliser output defined by

$\mathscr{2}[y(k)]=\arg \min _{s_{i, l} \in \mathscr{S}}\left|y(k)-s_{i, l}\right|^{2}$.

More precisely, at the symbol-spaced sample $k$, given $y(k)=\mathbf{w}_{\mathrm{c}}^{\mathrm{T}}(k) \mathbf{x}(k)+\mathbf{w}_{\mathrm{d}}^{\mathrm{T}}(k) \mathbf{x}(k)$ the CMA part adapts $\mathbf{w}_{\mathrm{c}}$ according to rule (13) by substituting $\mathbf{w}_{\mathrm{c}}$ for $\mathbf{w}$. The DD adaptation follows immediately after the CMA adaptation, but it only takes place if the CMA aided adjustment was deemed to be a successful one. Specifically, let $\tilde{y}(k)=\mathbf{w}_{\mathrm{c}}^{\mathrm{T}}(k+1) \mathbf{x}$ $(k)+\mathbf{w}_{\mathrm{d}}^{\mathrm{T}}(k) \mathbf{x}(k)$. Then the DD part adjusts $\mathbf{w}_{\mathrm{d}}$ according to

$$
\begin{aligned}
\mathbf{w}_{\mathrm{d}}(k+1)= & \mathbf{w}_{\mathrm{d}}(k)+\mu_{\mathrm{DD}} \delta(\mathscr{2}[\tilde{y}(k)]-\mathscr{2}[y(k)]) \\
& \times(\mathscr{2}[y(k)]-y(k)) \mathbf{x}^{*}(k),
\end{aligned}
$$

where $\mu_{\mathrm{DD}}$ is the adaptive gain of the DD STE and the indicator function is given by

$\delta(x)= \begin{cases}1, & x=0+j 0, \\ 0, & x \neq 0+j 0 .\end{cases}$

It can be seen that $\mathbf{w}_{\mathrm{d}}$ is updated only if the equaliser's hard decisions before and after the CMA aided adaptation are identical. A potential problem of (hard) DD adaptation is that if the decision is wrong, error propagation occurs, which subsequently degrades the adaptation. As analysed in [13] for the SISO case, if the STE's hard decisions before and after the CMA's adaptation are identical, the decision is likely to be a correct one. The DD adaptation, when it is safe to activate, has a significantly faster convergence speed over the pure CMA and is capable of reducing the steady-state MSE. The complexity of this concurrent CMA + DD scheme is summarised in Table 1. The adaptive gain $\mu_{\mathrm{DD}}$ invoked by the DD STE is typically chosen to be significantly larger than $\mu_{\mathrm{CMA}}$ of the CMA. Nevertheless, care must be exercised in choosing $\mu_{\mathrm{DD}}$ to avoid setting it to an excessively high value, which would inflict serious error propagation owing to incorrect decisions.

\subsection{Combined CMA and SDD scheme}

After steady-state equalisation is accomplished, the STEs soft output $y(k)$ may be approximated as $y(k) \approx s\left(k-k_{\mathrm{d}}\right)+v(k)$, where $k_{\mathrm{d}}$ is an unknown equaliser's decision delay, and $v(k)=$ $v_{R}(k)+\mathrm{j} v_{I}(k)$ may be approximated by a Gaussian white noise process. Thus, the STEs output may be reasonably accurately modelled by $M$ Gaussian clusters with the cluster means being $s_{i, l}$ for $1 \leqslant i, l \leqslant Q$. All the clusters have an approximate covariance of

$$
\left[\begin{array}{cc}
E\left[v_{R}^{2}(k)\right] & E\left[v_{R}(k) v_{I}(k)\right] \\
E\left[v_{I}(k) v_{R}(k)\right] & E\left[v_{I}^{2}(k)\right]
\end{array}\right] \approx\left[\begin{array}{cc}
\rho & 0 \\
0 & \rho
\end{array}\right] .
$$

Under the above conditions, the marginal PDF of $y(k)$ is approximately

$p(\mathbf{w}, y(k)) \approx \sum_{q=1}^{Q} \sum_{l=1}^{Q} \frac{p_{q, l}}{2 \pi \rho} \mathrm{e}^{-\left|y(k)-s_{q, l}\right|^{2} / 2 \rho}$,

where $p_{q, l}$ represents the a priori probabilities of $s_{q, l}$, $1 \leqslant q, l \leqslant Q$. The computation of PDF (19) involves the evaluation of $M \mathrm{e}^{\{\bullet\}}$ function values. However, a local approximation can be adopted for this PDF, which requires the evaluation of only four $\mathrm{e}^{\{\boldsymbol{\bullet}\}}$ function values. This is achieved by dividing the complex phasor plane into the $M / 4$ rectangular regions, as illustrated in Fig. 3. Each region $S_{i, l}$ contains four symbol points

$S_{i, l}=\left\{s_{p, q}, p=2 i-1,2 i, q=2 l-1,2 l\right\}$. 


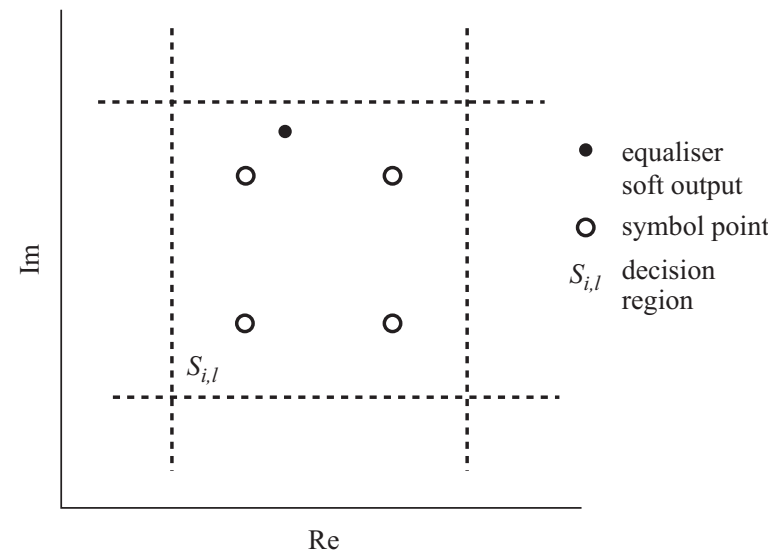

Fig. 3. Illustration of local decision regions for the soft decisiondirected adaptation procedure in a 64-QAM constellation.

If the STE's output $y(k) \in S_{i, l}$, a local approximation of the marginal PDF of $y(k)$ is given by

$\hat{p}(\mathbf{w}, y(k)) \approx \sum_{p=2 i-1}^{2 i} \sum_{q=2 l-1}^{2 l} \frac{1}{8 \pi \rho} \mathrm{e}^{-\left|y(k)-s_{p, q}\right|^{2} / 2 \rho}$,

where each a priori probability has been set to $\frac{1}{4}$. The combined CMA + SDD scheme operates a CMA STE and a SDD STE in unison. The CMA part is identical to that of the combined CMA + DD scheme. The task of this CMA equaliser is to open the eye, so that the local PDF expression (21) becomes approximately valid. The SDD STE is designed to maximise the log of the local marginal PDF criterion, i.e. the objective function of

$\bar{J}_{\mathrm{LMAP}}(\mathbf{w})=E\left[J_{\mathrm{LMAP}}(\mathbf{w}, y(k))\right]$,

where $J_{\text {LMAP }}(\mathbf{w}, y(k))=\rho \log (\hat{p}(\mathbf{w}, y(k)))$. Specifically, the SDD STE adapts $\mathbf{w}_{\mathrm{d}}$ according to

$\mathbf{w}_{\mathrm{d}}(k+1)=\mathbf{w}_{\mathrm{d}}(k)+\mu_{\mathrm{SDD}} \frac{\partial J_{\mathrm{LMAP}}(\mathbf{w}(k), y(k))}{\partial \mathbf{w}_{\mathrm{d}}}$,

where

$$
\begin{aligned}
& \frac{\partial J_{\text {LMAP }}(\mathbf{w}, y(k))}{\partial \mathbf{w}_{\mathrm{d}}} \\
& =\frac{\sum_{p=2 i-1}^{2 i} \sum_{q=2 l-1}^{2 l} \mathrm{e}^{-\left|y(k)-s_{p, q}\right|^{2} / 2 \rho}\left(s_{p, q}-y(k)\right)}{\sum_{p=2 i-1}^{2 i} \sum_{q=2 l-1}^{2 l} \mathrm{e}^{-\left|y(k)-s_{p, q}\right|^{2} 2 \rho}} \mathbf{x}^{*}(k)
\end{aligned}
$$

and $\mu_{\mathrm{SDD}}$ is an adaptive gain. The choice of $\rho$, defined in the context of the local PDF (21), should ensure a proper separation of the four clusters of
$S_{i, l}$. If the value of $\rho$ is too large, a desired degree of separation may not be achieved. On the other hand, if too small a $\rho$ value is used, the algorithm attempts to impose an overly tight control on the size of clusters and hence may fail to achieve its goal. Apart from these two extreme cases, the performance of the algorithm does not critically depend on the specific value of $\rho$. As the minimum distance between the two neighbouring constellation points is $2, \rho$ is typically chosen to be less than 1 .

Soft decision nature of the algorithm becomes explicit in (24), because rather than committing to a single hard decision $2[y(k)]$ as the DD scheme would, alternative decisions are also considered in the reduced size local region $S_{i, l}$ that includes $2[y(k)]$, and each tentative decision is weighted by an exponential term $\mathrm{e}^{[\bullet\}}$, which is a function of the distance between the STE's soft output $y(k)$ and the tentative decision $s_{p, q}$. This soft decision nature enables a simultaneous update of both $\mathbf{w}_{\mathrm{c}}$ and $\mathbf{w}_{\mathrm{d}}$ without a substantial risk of error propagation and, therefore, simplifies the associated operations. It also allows the potential employment of a larger adaptive gain $\mu_{\mathrm{SDD}}$, compared to the DD scheme. The complexity of the this CMA + SDD scheme is quantified in Table 1 . The four $\mathrm{e}^{\{\boldsymbol{\bullet}\}}$ function evaluations can be implemented with the aid of a lookup table in practice.

\section{Simulation study}

\subsection{Time-invariant system}

Two different performance criteria were used for assessing the convergence speed of the blind STE. The first one was the decision-based estimated MSE evaluated after each adaptation sample based on a block of $N_{\mathrm{MSE}}$ number of $T_{\mathrm{s}}$-spaced data samples, which is formulated as

$\mathrm{MSE}=\frac{1}{N_{\mathrm{MSE}}} \sum_{k=1}^{N_{\mathrm{MSE}}}|2[y(k)]-y(k)|^{2}$,

and the second one was the maximum distortion (MD) measure defined as

$\mathrm{MD}=\frac{\sum_{i=0}^{N_{f}-1}\left|f_{i}\right|-\left|f_{i_{\max }}\right|}{\left|f_{i_{\max }}\right|}$,

where $\mathbf{f}=\left[\begin{array}{llll}f_{0} & f_{1} & \cdots & f_{N_{f}-1}\end{array}\right]^{\mathrm{T}}$ was the combined impulse response of the channels and STE defined by

$\mathbf{f}=\sum_{l=1}^{L} \overline{\mathbf{w}}_{l}^{\mathrm{o}} \star \overline{\mathbf{a}}_{l}^{\mathrm{e}}+\overline{\mathbf{w}}_{l}^{\mathrm{e}} \star \overline{\mathbf{a}}_{l}^{\mathrm{o}}$, 
Table 2

$T_{\mathrm{s}} / 2$-Spaced CIRs for the simulated time-invariant SIMO system

\begin{tabular}{lrrrr}
\hline$l$ & \multicolumn{1}{l}{$\overline{\mathbf{a}}_{l}$} & & & \\
\hline 1 & $-0.2+j 0.3$ & $-0.5+j 0.4$ & $0.7-j 0.6$ & $0.2+j 0.1$ \\
2 & $0.3+j 0.2$ & $0.7-j 0.5$ & $-0.5+j 0.4$ & $0.4+j 0.3$ \\
3 & $-0.1-j 0.2$ & $0.6+j 0.4$ & $-0.4+j 0.3$ & $0.1+j 0.3$ \\
4 & $0.6+j 0.7$ & $0.5+j 0.4$ & $-0.1-j 0.2$ & $0.2+j 0.1$ \\
\hline
\end{tabular}

with $\star$ denoting convolution and $N_{f}=N_{c}+m-1$ being the length of the $T_{\mathrm{s}}$-spaced combined impulse response, and $f_{i_{\max }}=\max \left\{f_{i}, 0 \leqslant i \leqslant N_{f}-1\right\}$. The STE's output signal constellation after convergence was also shown using $N_{\text {test }}=6000$ number of $T_{\mathrm{s}^{-}}$ spaced data samples not used in the adaptation phase.

In our simulation, $L=$ four receiver antennas were employed and the transmitted symbol sequence was of 256-QAM format. The four $T_{\mathrm{s}} / 2-$ spaced propagation channels encountered by the four different received signals were listed in Table 2 . The actual simulated channels were normalised according to $\overline{\mathbf{a}}_{l} /\left\|\overline{\mathbf{a}}_{l}\right\|$ for $1 \leqslant l \leqslant 4$ in order to ensure that each channel had a unit energy. The noise variance was $\sigma_{e}^{2}=0.00425$, yielding a SNR of $40 \mathrm{~dB}$. The length $2 m$ of the $T_{\mathrm{s}} / 2$-spaced temporal filters should be chosen to be sufficiently long for opening the initially ISI contaminated closed eye and for obtaining a good steady-state performance. However, the STE must not be excessively long, since that would cause problems in terms of seriously enhancing the noise and reduce the convergence speed. The actual value of $2 m$ used in our simulations was found empirically and was set to $2 m=10$. The block length for estimating MSE (25) was set to $N_{\mathrm{MSE}}=1000$. The values of the various adaptive algorithmic parameters, namely the adaptive gains required by the CMA, DD and SDD schemes, as well as the width for the SDD, were also chosen empirically for the sake of ensuring both fast convergence and good steadystate performance.

Because this was a high-order 256-QAM system, for the CMA blind adaptive algorithm, the adaptive gain had to be chosen to be sufficiently small for the sake of avoiding divergence and $\mu_{\mathrm{CMA}}=10^{-8}$ was used. For the CMA + DD, the two adaptive gains were chosen to be $\mu_{\mathrm{CMA}}=10^{-8}$ and $\mu_{\mathrm{DD}}=4 \times$ $10^{-5}$. Any further increase of $\mu_{\mathrm{DD}}$ used for the DD adaptation process would degrade the attainable a

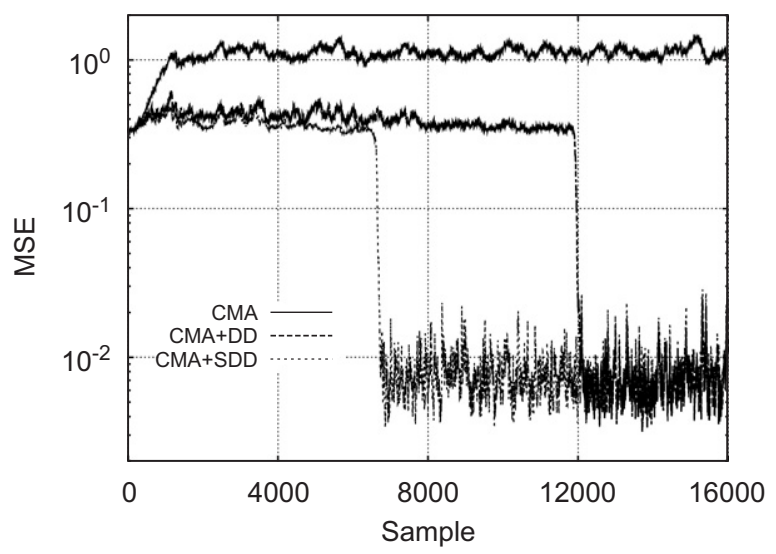

b

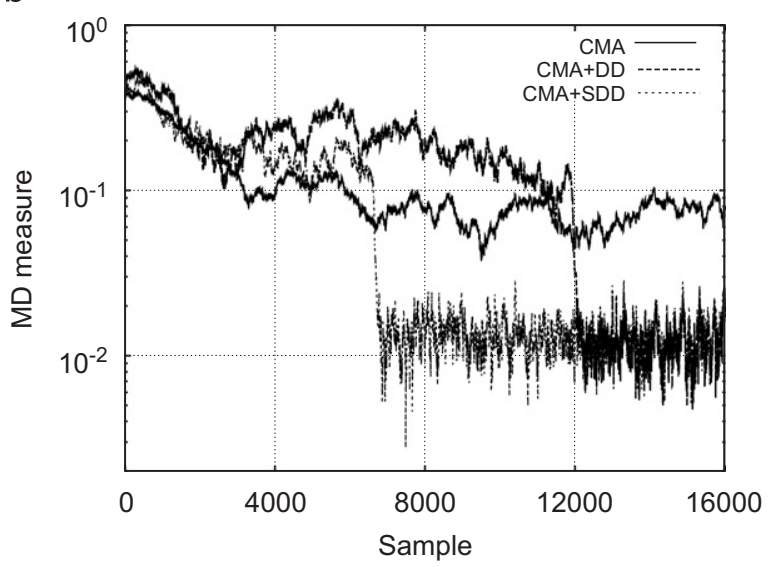

Fig. 4. Comparison of convergence performance in terms of: (a) estimated MSE and (b) MD measure for the time-invariant CIRs of Table 2 with 256-QAM format and at an SNR of $40 \mathrm{~dB}$.

performance significantly due to the effects of error propagation. As for the $\mathrm{CMA}+\mathrm{SDD}$, the two adaptive gains were set to $\mu_{\mathrm{CMA}}=10^{-8}$ and $\mu_{\mathrm{SDD}}=5 \times 10^{-5}$, while the width parameter was chosen to be $\rho=0.6$. The learning curves of the three blind STEs, namely the CMA, CMA + DD and CMA + SDD, quantified in terms of the estimated MSE and MD measures, are depicted in Fig. 4(a) and (b), respectively, while the equaliser's output signal constellations after convergence are illustrated in Fig. 5(a)-(c), respectively. The results clearly demonstrate that both the CMA + DD and the CMA + SDD schemes have the same steadystate equalisation performance, which is significantly better than the performance achieved by the pure CMA. It can also be seen that the CMA + SDD STE has a significantly faster convergence 
a

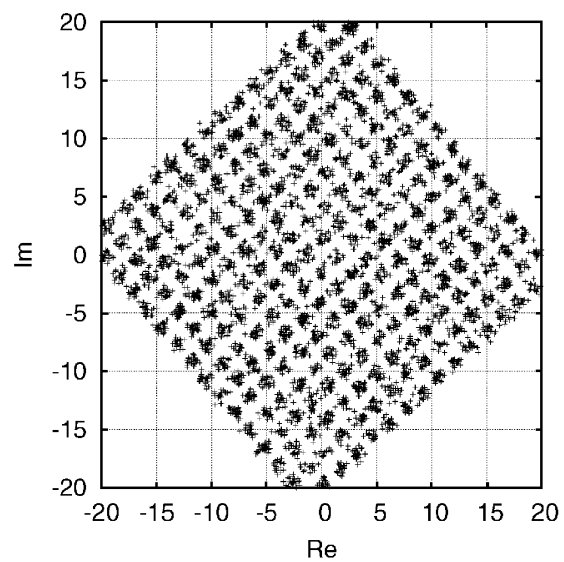

C

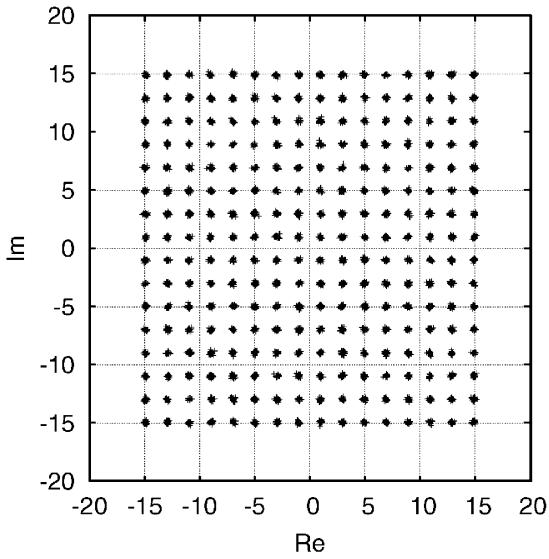

b

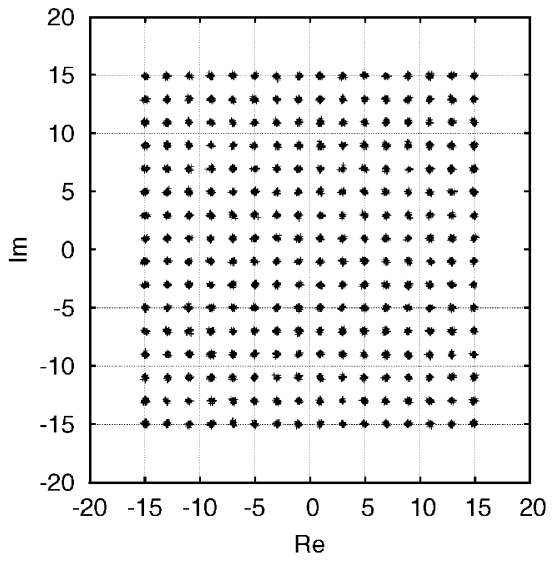

d

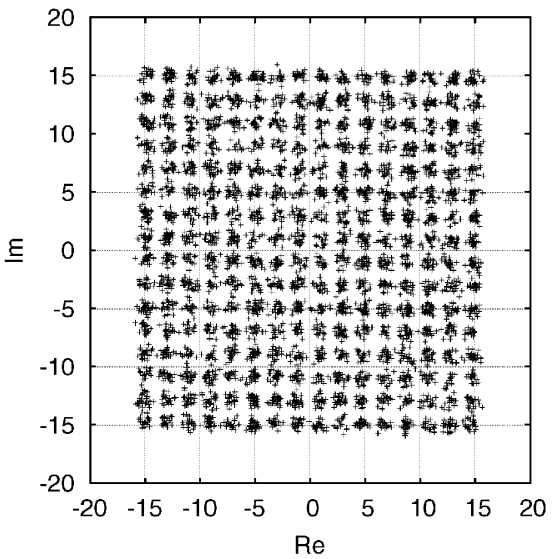

Fig. 5. Space-time equaliser's output signal constellations after convergence: (a) the CMA, (b) the CMA + DD, (c) CMA + SDD and (d) the CMA with the $48^{\circ}$ phase compensation for the time-invariant CIRs of Table 2 with 256-QAM format and at an SNR of $40 \mathrm{~dB}$.

speed than the CMA + DD STE. Observing the learning curves in Fig. 4(b), it can clearly be seen that the effect of error propagation inflicted upon the DD adaptation is significantly more grave than that imposed on the SDD scheme.

The CMA-based STE's output signal constellation depicted in Fig. 5(a) after convergence exhibits a $\left(48^{\circ}\right)$ phase shift, while Fig. $5(\mathrm{~d})$ shows the CMA STE's output signal constellation after a $48^{\circ}$ phase compensation. During the blind adaptation procedure, this phase shift is unknown to the CMA. However, if we assume that this phase shift is known to the adaptive algorithm, the convergence of the CMA quantified in terms of the estimated MSE could be improved. Fig. 6 depicts the MSE convergence performance of the pure CMA benefitting from the $48^{\circ}$ phase compensation. Even with the benefit of this phase compensation, the steadystate MSE of the CMA STE is considerably inferior

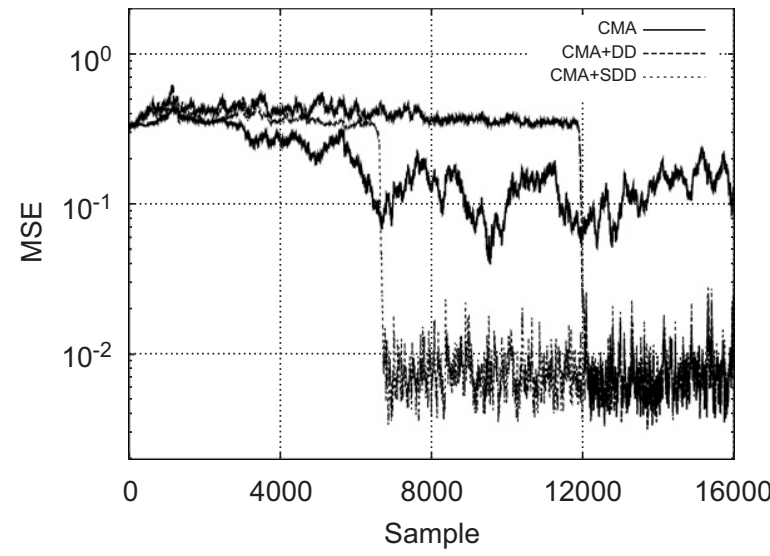

Fig. 6. Comparison of convergence performance in terms of the estimated MSE for the time-invariant CIRs of Table 2 with 256QAM format and at an SNR of $40 \mathrm{~dB}$. The $48^{\circ}$ phase shift of the pure CMA STE is assumed to be known and has been compensated. No phase compensation is performed for the CMA part of the CMA-DD and CMA-SDD STEs. 
to those of the CMA + DD and CMA + SDD schemes.

\subsection{Fading system}

Again, $L=$ four receiver antennas were used but the transmitted symbol sequence was of 64-QAM format at the symbol rate of $4 \mathrm{MHz}$. As usual, a baseband system was simulated. The transmission pulse had a raised-cosine shape associated with a rolloff factor 0.5 and a square root shaped Nyquist characteristic was implemented at both the transmitter and receiver. The fading channels were implemented using the following tap-delay-line models for $1 \leqslant l \leqslant 4$ :

$v_{l}(t)=c_{0, l}(t) u(t)+c_{1, l}(t) u\left(t-\tau_{1}\right)+c_{2, l}(t) u\left(t-\tau_{2}\right)$,

a

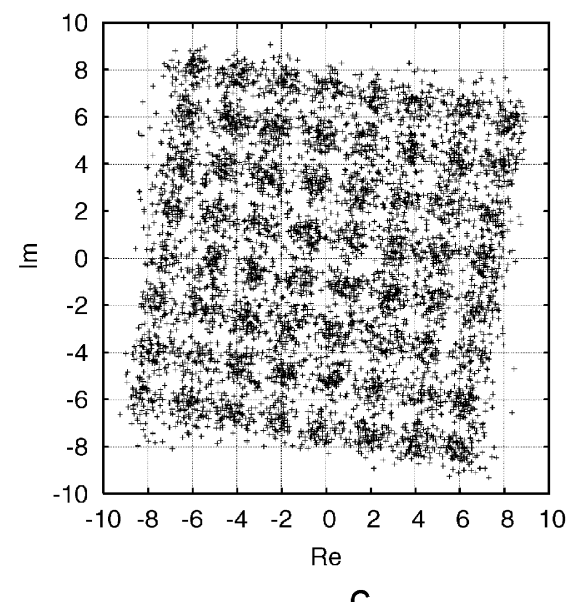

where $u(t)$ was the transmitter's output, $v_{l}(t)$ was the lth fading channel's output, $\tau_{1} \approx 0.44 T_{\mathrm{s}}$ and $\tau_{2} \approx 1.13 T_{\mathrm{s}}$. The magnitudes of the complex-valued tap weights $c_{i, l}(t), 0 \leqslant i \leqslant 2$, were i.i.d. Rayleigh processes, while the associated root mean powers of $c_{i, l}(t)$ were $[0.7+j 0.70 .6+j 0.60 .5+j 0.5]$, for $0 \leqslant i \leqslant 2$, respectively. Continuously fluctuating fading was used at a fading rate of $40 \mathrm{~Hz}$, which provided a different fading magnitude and phase for each transmitted symbol. The normalised Doppler frequency for the simulated system was $10^{-5}$. The noise powers were $\sigma_{e}^{2}=0.011$, yielding an average SNR of $30 \mathrm{~dB}$. In order to simulate highly correlated SIMO channels that are commonly encountered in practice, the spatial covariance matrix generated for the scenario of a single transmitter antenna radiating signals to four receiver antennas was

b
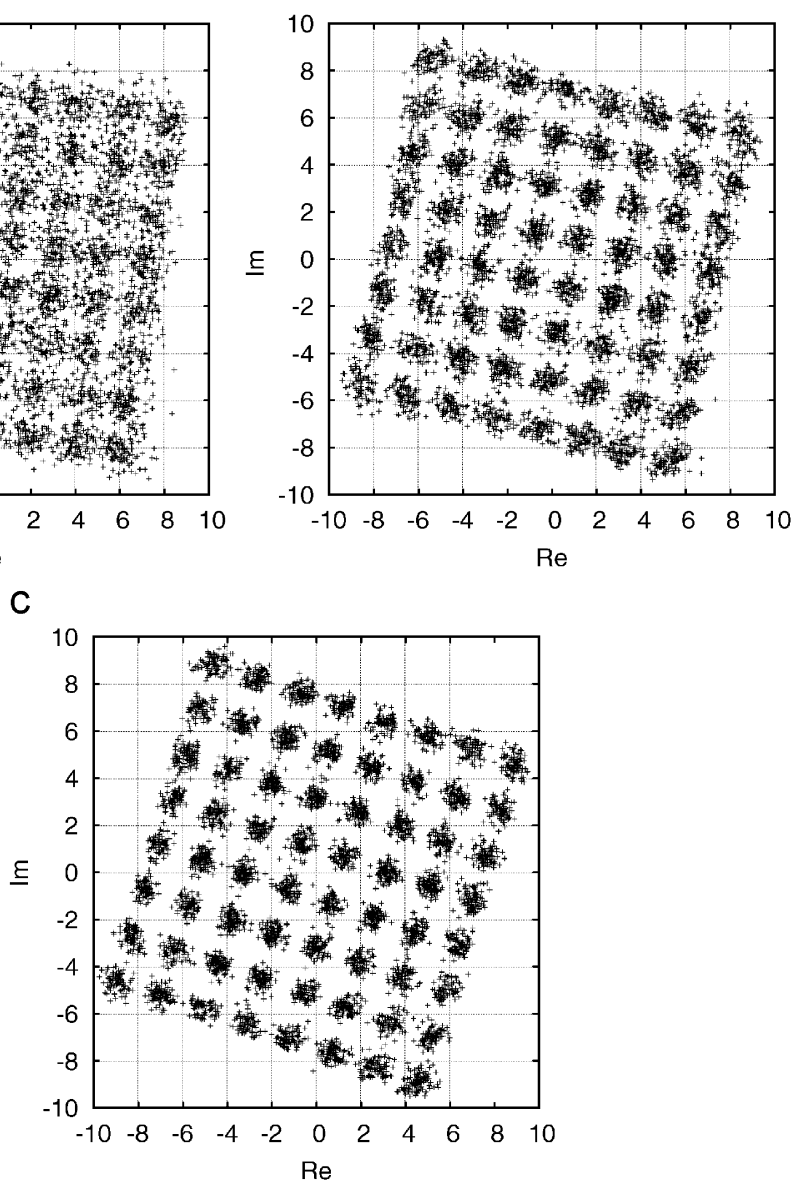

Fig. 7. CMA equaliser's output signal constellations for the fading system with 64-QAM format and at an average SNR of $30 \mathrm{~dB}$ after adaptation using: (a) 8000 symbols, (b) 12000 symbols, and (c) 16000 symbols. A total of $6000 T_{\text {s}}$-spaced samples were used for portraying the signal constellation invoking continuous adaptation. 
represented by

$\mathbf{R}=\left[\begin{array}{cccc}1 & \alpha & \beta & \gamma \\ \alpha^{*} & 1 & \alpha & \beta \\ \beta^{*} & \alpha^{*} & 1 & \alpha \\ \gamma^{*} & \beta^{*} & \alpha^{*} & 1\end{array}\right]$,

with $\alpha=0.97 \mathrm{e}^{-j 0.8}, \beta=0.94 \mathrm{e}^{-j 1.6}$ and $\gamma=0.88 \mathrm{e}^{-j 2.4}$ [29]. Let $\mathbf{R}=\mathbf{Q} \mathbf{\Sigma} \mathbf{Q}^{H}$, where $\mathbf{Q}$ consists of the eigenvectors associated with $\mathbf{R}$ and $\boldsymbol{\Sigma}$ is diagonal, whose diagonal elements are the eigenvalues of $\mathbf{R}$. Then the receiver antenna outputs are represented by

$\left[\begin{array}{l}x_{1}(t) \\ x_{2}(t) \\ x_{3}(t) \\ x_{4}(t)\end{array}\right]=\mathbf{Q} \Sigma^{1 / 2}\left[\begin{array}{l}v_{1}(t) \\ v_{2}(t) \\ v_{3}(t) \\ v_{4}(t)\end{array}\right]+\left[\begin{array}{l}e_{1}(t) \\ e_{2}(t) \\ e_{3}(t) \\ e_{4}(t)\end{array}\right]$.

a

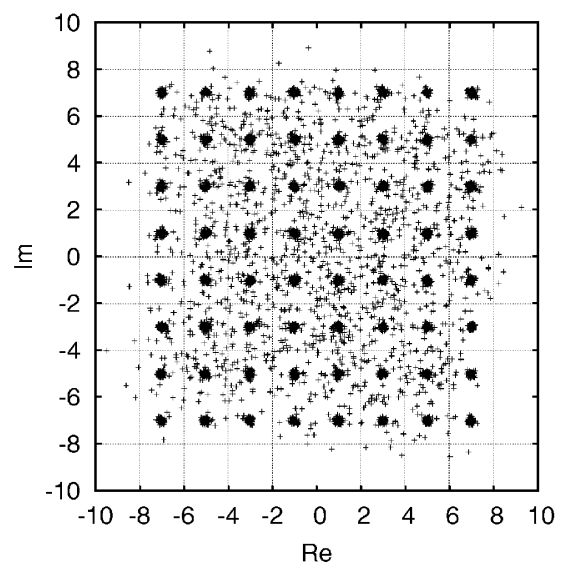

C
The receiver outputs were sampled at a rate of twice the symbol rate and passed to a STE whose $T_{\mathrm{s}} / 2$ spaced temporal filters had an order of 10.

The appropriate adaptive algorithmic parameters were found empirically, which were $\mu_{\mathrm{CMA}}=$ $2 \times 10^{-8}$ for the CMA; $\mu_{\mathrm{CMA}}=2 \times 10^{-8}$ and $\mu_{\mathrm{DD}}=5 \times 10^{-5}$ for the CMA + DD; and $\mu_{\mathrm{CMA}}$ $=2 \times 10^{-8}, \quad \mu_{\mathrm{SDD}}=10^{-4}$ and $\rho=0.5$ for the $\mathrm{CMA}+\mathrm{SDD}$. Note that using $\mu_{\mathrm{CMA}}=5 \times 10^{-8}$ would force the CMA to diverge, while choosing $\mu_{\mathrm{DD}}=10^{-4}$ would result in divergence for the DD adaptation. Fig. 7 depicts the pure CMA STE's output signal constellations after: (a) adaptation using 8000 symbols, (b) adaptation employing 12000 symbols, and (c) adaptation invoking 16000 symbols. Each signalconstellation was shown for $6000 T_{\mathrm{s}}$-spaced samples invoking continuous i.e. symbol-by-symbol-based adaptation, since the chan-

b

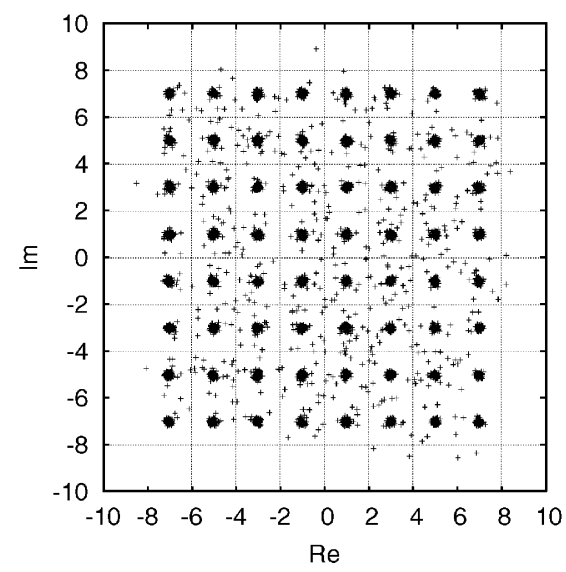

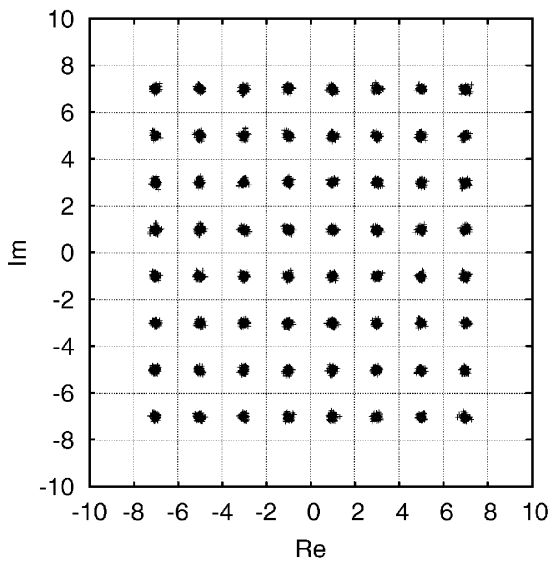

Fig. 8. CMA + DD equaliser's output signal constellations for the fading system with 64-QAM format and at an average SNR of $30 \mathrm{~dB}$ after adaptation using: (a) 4000 symbols, (b) 5000 symbols, and (c) 6000 symbols. A total of $6000 T_{\mathrm{s}}$-spaced samples were used for portraying the signal constellation invoking continuous adaptation. 
a

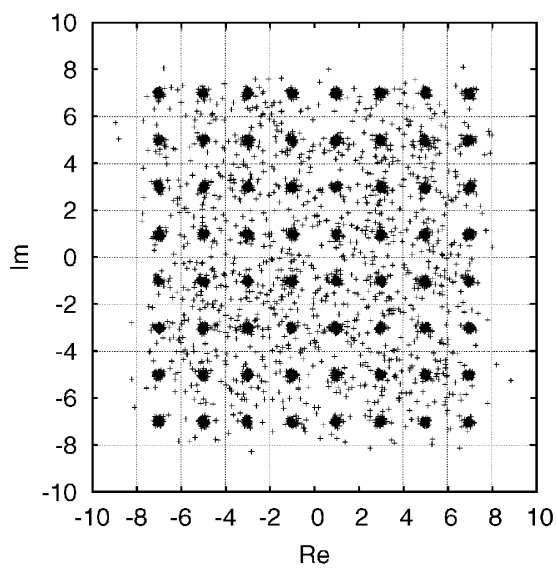

b

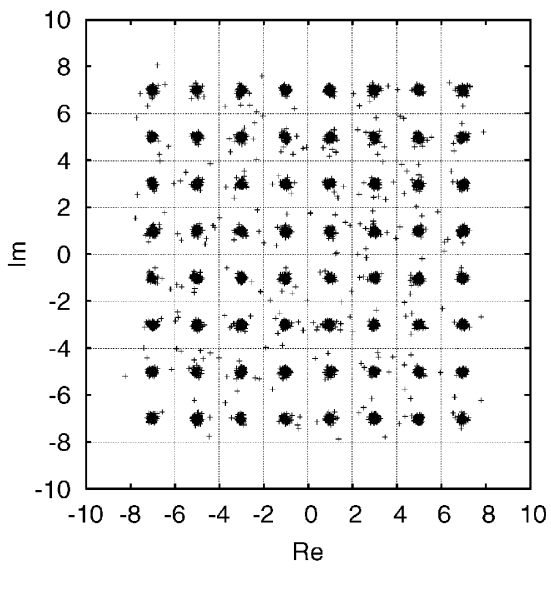

C

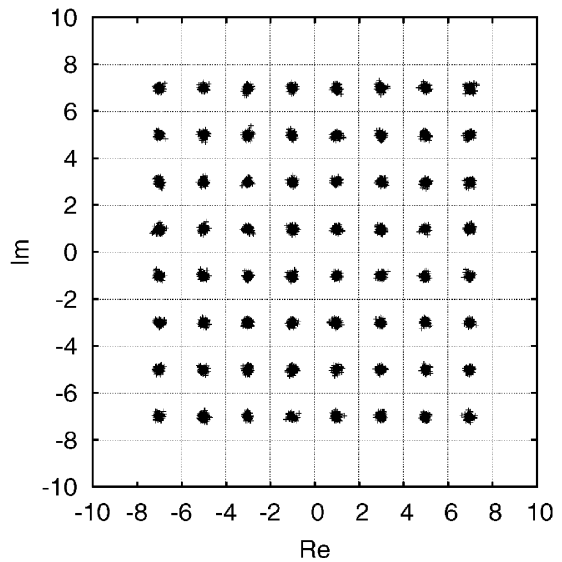

Fig. 9. CMA + SDD equaliser's output signal constellations for the fading system with 64-QAM format and at an average SNR of $30 \mathrm{~dB}$ after adaptation using: (a) 2500 symbols, (b) 3500 symbols, and (c) 4500 symbols. A total of $6000 T_{\mathrm{s}}$-spaced samples were used for portraying the signal constellation invoking continuous adaptation.

nels were time-varying. Fig. 8 plots the CMA + DD STE's output signal constellations after: (a) adaptation using 4000 symbols, (b) adaptation assisted by 5000 symbols, and (c) adaptation aided by 6000 symbols. Similarly, Fig. 9 shows the CMA + SDD STE's output signal constellations after: (a) adaptation invoking 2500 symbols, (b) adaptation employing 3500 symbols, and (c) adaptation using 4500 symbols. It is readily seen that the CMA + SDD scheme had a significantly faster convergence than the CMA + DD arrangement. Because this was a time varying system, the performance criteria of (25) and (26) could not be evaluated. To provide an overall convergence picture, we adopted a simple moving average-based estimate for the MSE, defined by

$\tilde{\varepsilon}^{2}(k)=\eta \times \tilde{\varepsilon}^{2}(k-1)+(1-\eta) \times \frac{1}{2}|2[y(k)]-y(k)|^{2}$.
Fig. 10 compares the convergence performance of the three blind STEs using this moving average-based MSE estimate, with the value of $\eta$ set to 0.95 . From Fig. 7, it is seen that the CMA-based STE exhibited a $17^{\circ}$ phase shift. The MSE estimate (31) plotted in Fig. 10 for the CMA-based STE was obtained by compensating this $17^{\circ}$ phase shift.

\section{Conclusions}

In this paper, blind space-time equalisation has been proposed and characterised in the context of SIMO systems employing multiple receiver antennas. Emphasis has been put to SIMO systems that use bandwidth efficient highthroughput QAM schemes. A novel low-complexity blind STE has been proposed based on combining a CMA equaliser and a SDD equaliser. The 


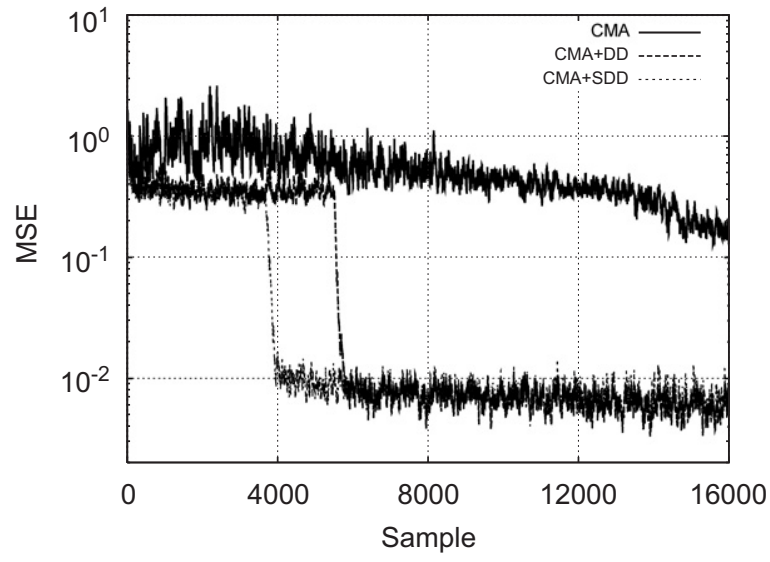

Fig. 10. Comparison of convergence performance in terms of the estimated MSE for the fading system with 64-QAM format and at an average SNR of $30 \mathrm{~dB}$. The $17^{\circ}$ phase shift of the pure CMA STE is assumed to be known and has been compensated. No phase compensation is performed for the CMA part of the CMA-DD and CMA-SDD STEs.

simulation results provided have demonstrated that this blind STE performs substantially better than its individual components. Our future research may consider similar multiple-transmitter arrangements.

\section{References}

[1] G.J. Foschini, Layered space-time architecture for wireless communication in a fading environment when using multiple antennas, Bell Labs Tech. J. 1 (2) (1996) 41-59.

[2] J.H. Winters, Smart antennas for wireless systems, IEEE Pers. Commun. 5 (1) (1998) 23-27.

[3] A.J. Paulraj, B.C. Ng, Space-time modems for wireless personal communications, IEEE Pers. Commun. 5 (1) (1998) 36-48.

[4] A. Paulraj, R. Nabar, D. Gore, Introduction to Space-Time Wireless Communications, Cambridge University Press, Cambridge, UK, 2003.

[5] L. Hanzo, L.-L. Yang, E.-L. Kuan, K. Yen, Single- and Multi-Carrier DS-CDMA: Multi-User Detection, SpaceTime Spreading, Synchronisation, Standards and Networking, IEEE Press-Wiley, New York, 2003.

[6] T. Tang, R.W. Heath Jr., Space-time interference cancellation in MIMO-OFDM systems, IEEE Trans. Veh. Technol. 54 (5) (2005) 1802-1816.

[7] L. Hanzo, S.X. Ng, T. Keller, W. Webb, Quadrature Amplitude Modulation: From Basics to Adaptive TrellisCoded, Turbo-Equalised and Space-Time Coded OFDM, CDMA and MC-CDMA Systems, Wiley and IEEE Press, Chichester, UK, 2004.
[8] D. Godard, Self-recovering equalization and carrier tracking in two-dimensional data communication systems, IEEE Trans. Commun. COM-28 (1980) 1867-1875.

[9] J.R. Treichler, B.G. Agee, A new approach to multipath correction of constant modulus signals, IEEE Trans. Acoust. Speech Signal Process. ASSP-31 (2) (1983) 459-472.

[10] J.R. Treichler, Application of blind equalization techniques to voiceband and RF modems, in: Preprints 4th IFAC International Symposium Adaptive Systems in Control and Signal Processing (France), 1992, pp. 705-713.

[11] N.K. Jablon, Joint blind equalization, carrier recovery, and timing recovery for high-order QAM signal constellations, IEEE Trans. Signal Process. 40 (6) (1992) 1383-1398.

[12] O. Macchi, E. Eweda, Convergence analysis of selfadaptive equalizers, IEEE Trans. Inf. Theory IT-3 (2) (1984) 161-176.

[13] F.C.C. De Castro, M.C.F. De Castro, D.S. Arantes, Concurrent blind deconvolution for channel equalization, in: Proceedings of the ICC'2001 (Helsinki, Finland), June 11-15, 2001, vol. 2, pp. 366-371.

[14] A. Benveniste, M. Goursat, Blind equalizers, IEEE Trans. Commun. COM-32 (8) (1984) 871-883.

[15] S.J. Nowlan, G.E. Hinton, A soft decision-directed LMS algorithm for blind equalization, IEEE Trans. Commun. 41 (2) (1993) 275-279

[16] J. Karaoguz, S.H. Ardalan, A soft decision-directed blind equalization algorithm applied to equalization of mobile communication channels, in: Proceedings of the ICC'1992 (Chicago, USA), 1992, vol. 3, pp. 343.4.1-343.4.5.

[17] S. Chen, S. McLaughlin, P.M. Grant, B. Mulgrew, Multistage blind clustering equaliser, IEEE Trans. Commun. 43 (3) (1995) 701-705.

[18] S. Chen, E.S. Chng, Concurrent constant modulus algorithm and soft decision directed scheme for fractionally-spaced blind equalization, in: Proceedings of the ICC 2004 (Paris, France), June 20-24, 2004, vol. 4, pp. 2342-2346.

[19] H. Gazzah, P.A. Regalia, J.-P. Delmas, Asymptotic eigenvalue distribution of block Toeplitz matrices and application to blind SIMO channel identification, IEEE Trans. Inf. Theory. 47 (3) (2001) 1243-1251.

[20] D. Luengo, I. Santamaria, J. Ibanez, L. Vielva, C. Pantaleon, A fast blind SIMO channel identification algorithm for sparse sources, IEEE Signal Process. Lett. 10 (5) (2003) 148-151.

[21] I. Santamaria, J. Via, C.C. Gaudes, Robust blind identification of SIMO channels: a support vector regression approach, in: Proceedings of the ICASSP'2004, 17-21 May, 2004, vol. 5, pp. 673-676.

[22] A. Ranheim, A decoupled approach to adaptive signal separation using an antenna array, IEEE Trans. Veh. Technol. 48 (3) (1999) 676-682.

[23] A. Dogandzic, A. Nehorai, Generalized multivariate analysis of variance-A unified framework for signal processing in correlated noise, IEEE Signal Process. Mag. 20 (5) (2003) $39-54$.

[24] J.J. Shynk, R.P. Gooch, The constant modulus array for cochannel signal copy and direction finding, IEEE Trans. Signal Process. 44 (3) (1996) 652-660.

[25] J.J. Shynk, A.V. Keerthi, A. Mathur, Steady-state analysis of the multistage constant modulus array, IEEE Trans. Signal Process. 44 (4) (1996) 948-962. 
[26] H.H. Zeng, L. Tong, C.R. Johnson Jr., Relationships between the constant modulus and Wiener receivers, IEEE Trans. Inf. Theory 44 (4) (1998) 1523-1538.

[27] K. Yang, T. Ohira, Y. Zhang, C.-Y. Chi, Super-exponential blind adaptive beamforming, IEEE Trans. Signal Process. 52 (6) (2004) 1549-1563.
[28] R. Johnson Jr., P. Schniter, T.J. Endres, J.D. Behm, D.R. Brown, R.A. Casas, Blind equalization using the constant modulus criterion: a review, Proc. IEEE 86 (10) (1998) 1927-1950.

[29] 3GPP, Tx diversity solutions for multiple antennas, 3GPP TSG RAN WG1 document TR25.869 V0.1.1, November 2001. 